\title{
Crecimiento y distribución de biomasa aérea en clones de castaño híbrido a edad temprana
}

García-Villabrille, J.D. ${ }^{1}$, Arias-Vázquez, O. ${ }^{2}$, Ferradás García, H. ${ }^{1}$, Gorgoso-Varela, J.J. ${ }^{3}$, Rojo-Alboreca, A. ${ }^{*}$

${ }^{1}$ Unidade de Xestión Forestal Sostible, Departamento de Enxeñaría Agroforestal, Escola Politécnica Superior, Universidade de Santiago de Compostela, C/ Benigno Ledo s/n, Campus Universitario, 27002 Lugo.

${ }^{2}$ BOIBEL Forestal S.L., Pazo de Galegos, 15686 Frades, A Coruña.

${ }^{3}$ Departamento de Biología de Organismos y Sistemas, Escuela Politécnica de Mieres, Universidad de Oviedo. C/ Gonzalo Gutiérrez Quirós s/n., 33600 Mieres, Asturias.

*Autor de correspondencia e-mail: alberto.rojo@usc.es

\section{Resumen}

Se presentan los resultados de la medición en 228 ejemplares de castaños híbridos de 20 clones distintos en vivero, de edades comprendidas entre 4 y 7 años. Se realizaron muestreos destructivos para caracterizar las principales potencialidades de los clones en lo que se refiere a crecimiento y distribución de la biomasa aérea (fuste y ramas). Así, se elaboró una tarifa de biomasa total (peso seco), conjunta para todos los clones, en cuyo ajuste las mejores variables fueron el diámetro en la base y el diámetro normal, con $R^{2}=0,87$ y REMC $=0,61 \mathrm{~kg}$ árbol ${ }^{-1}$. Además, se establecieron unos rankings de clones según su capacidad para obtener:

— Una mayor capacidad de ramificación (medida mediante la biomasa de ramas), por su influencia en la aptitud para producción de fruto.

— Una mayor producción de biomasa del fuste y del crecimiento en diámetro y altura, por su influencia en la aptitud para uso forestal maderable.

Palabras clave: Castanea, fuste, ramas, tarifa biomasa. 


\section{Introducción}

El castaño, Castanea sativa, es un árbol caducifolio corpulento, capaz de alcanzar los 35 metros de altura y los $150 \mathrm{~cm}$ de diámetro; además, tiene una excelente capacidad para el rebrote de cepa y, en consecuencia, para el cultivo en monte bajo. Hasta fechas recientes las actividades de selección realizadas por investigadores europeos se han centrado en la descripción de variedades de fruto a partir de las selecciones tradicionales, así como en la hibridación interespecífica para incrementar la resistencia a las dos enfermedades más importantes: la tinta y el chancro (FernándezLópez y Alía, 2008).

La especie más utilizada para hibridación con castaño europeo ha sido el castaño japonés (Castanea crenata) y, en menor medida, el castaño chino (Castanea mollisima). Los híbridos mejoran la resistencia y el vigor y crecen muy bien en climas húmedos con poca oscilación térmica. Sin embargo, son más sensibles a la sequía estival que Castanea sativa, y su brotación temprana los hace más sensibles a las heladas de primavera (Fernández-López y Alía, 2008).

Álvarez-Álvarez et al. (2000) comentan que la obtención de planta resistente a la enfermedad de la tinta está conseguida en los castaños híbridos, pero que todavía no se han logrado plantas resistentes a la enfermedad del chancro, lo cual es objeto de investigación. El problema que se plantea cuando se utiliza este material híbrido resistente a la tinta es la ausencia de información sobre su adaptación ecológica a los lugares de plantación.

En cuanto a su aptitud, aunque antiguamente los clones comercializados por la Administración Pública se vendían con un reducido nivel de información en este sentido, en la actualidad los viveros privados manejan algunos clones híbridos con potencialidades conocidas. Álvarez-Álvarez et al. (2004) publicaron una tabla de aptitudes de cada uno de los clones que se encontraban en el vivero de A Famelga (A Coruña). Fernández-López y Miranda (2007), como resultado de 18 años de estudios, facilitaron que la Consellería de Medio Rural de la Xunta de Galicia publicase la Orden (de 16 de abril de 2007) por la que se aprobaba la delimitación y la determinación de los materiales de base para la producción de materiales forestales de reproducción de varias especies en Galicia, incluyendo los distintos clones artificiales del género Castanea (Xunta de Galicia, 2007).

El objetivo fundamental del presente estudio consistió en analizar el crecimiento y la distribución de la biomasa aérea (fuste y ramas) en clones de castaño híbrido a edad temprana. Para ello, a partir de la medición en vivero de una muestra de árboles menores de 7 años se pretendió establecer unos rankings de clones de castaño híbrido en función de los valores de las siguientes variables, que condicionan (aunque no sea completamente) algunas de las aptitudes comúnmente buscadas:

- Mayor capacidad de ramificación (medida mediante la biomasa de ramas), por su influencia en la aptitud para producción de fruto.

- Mayor producción de biomasa del fuste y del crecimiento en diámetro y altura, por su influencia en la aptitud para uso forestal maderable.

Por otro lado, resulta de interés contar con ecuaciones de estimación de la bioma- 
sa, especialmente en el caso de árboles de pequeñas dimensiones, en los que la variabilidad de la altura de copa (especialmente cuando la ramificación principal empieza debajo de 1,3 m) afecta a la medición del diámetro normal, que es la variable usualmente utilizada en estas ecuaciones. Por esta razón, en este trabajo también se ajustó una tarifa de biomasa total seca, de árbol individual, para árboles de pequeñas dimensiones.

\section{Material y métodos}

Los datos correspondientes a los clones estudiados se obtuvieron en un vivero de la localidad de Frades (provincia de A Coruña) entre los años 2012 y 2015 . El clima de la zona es templado, con una temperatura media de $11-12^{\circ} \mathrm{C}$ y una precipitación media anual de 1300-1400 mm (datos obtenidos de la estación meteorológica de Olas, A Coruña). Aunque el vivero se dedica a la producción de diversas especies, la mayor parte de su superficie está destinada al engorde y recepe de una amplia variedad de clones de castaño.

La información recogida en cada árbol fue la siguiente: edad $(t$, años) por conteo de anillos; diámetro en la base $\left(d_{b}, \mathrm{~cm}\right)$ y diámetro normal $\left(d_{n}, \mathrm{~cm}\right)$, medidos con calibre milimetrado; altura total $(h, \mathrm{~m})$ y altura de fuste $(h, \mathrm{~m})$, medidas con cinta métrica de precisión a $0.01 \mathrm{~m}$; peso verde de fuste $\left(p v f, \mathrm{~kg}^{2}\right.$ árbol $\left.^{-1}\right)$, peso verde de ramas $\left(p v_{r}, \mathrm{~kg}\right.$ árbol $\left.^{-1}\right)$, peso seco de fuste $\left(w_{f}, \mathrm{~kg}\right)$ y peso seco de ramas $\left(w_{r}, \mathrm{~kg}\right)$, medidos con báscula de campo de precisión $0.2 \mathrm{~kg}$.

En total, para en este trabajo se midieron 228 ejemplares de castaños híbridos de 20 clones distintos, con edades comprendidas entre 4 y 7 años. La selección de los árboles a cortar se basó exclusivamente en la disponibilidad de los ejemplares en el vivero y en la oportunidad de cortarlos antes de su recepe comercial. En la Tab. 1 se muestra la relación de los clones utilizados y el número de individuos medidos de cada uno de ellos, junto como un resumen de sus principales variables.

La muestra que se empleó en cada árbol para la estimación de biomasa correspondió a dos discos, que delimitan el fuste, y el $20 \%$ en peso de las ramas de todos los grosores. La rodaja inferior del fuste fue tomada a $20 \mathrm{~cm}$ del suelo, donde se practicó un corte en bisel para el posterior recepado del árbol, y la rodaja superior del fuste se extrajo por debajo del punto de aparición de la copa. El grosor máximo de ramas fue de $2.5 \mathrm{~cm}$, haciendo un grupo único con todas ellas. El secado se realizó en estufa a $103^{\circ} \mathrm{C} \pm 0.5$ hasta pesada constante. Las pesadas en laboratorio se hicieron con báscula de precisión hasta $0.01 \mathrm{~g}$.

Se realizó un análisis de relaciones entre variables por métodos gráficos y una selección previa de variables para el modelo de biomasa mediante regresión por pasos. Este análisis fue complementado por el cálculo del número de condición o IC (Myers, 1986) a partir de modelos linealizados, asumiendo que valores inferiores a 30 no indican problemas de colinealidad (Belsley, 1991). El ajuste del modelo de biomasa se basó en el modelo de Schumacher and Hall (1933), y se realizó por mínimos cuadrados con regresión ponderada (GLS), empleando el software R (R Core Team, 2014). 
Tabla 1. Número de pies de cada clon y resumen de sus principales variables dendrométricas.

\begin{tabular}{|c|c|c|c|c|c|c|c|}
\hline Clon & N $^{\text {pies }}$ & $\begin{array}{c}\text { Edad } \\
\text { (años) }\end{array}$ & $\begin{array}{c}\text { Diámetro de } \\
\text { la base }(\mathrm{cm})\end{array}$ & $\begin{array}{c}\text { Diámetro } \\
\text { normal }(\mathrm{cm})\end{array}$ & $\begin{array}{c}\text { Altura } \\
\text { fuste }(\mathrm{m})\end{array}$ & $\begin{array}{c}\text { Altura } \\
\text { total }(\mathrm{m})\end{array}$ & $\begin{array}{c}\text { Biomasa total } \\
\text { o peso seco (kg) }\end{array}$ \\
\hline 3 & 3 & 6 & 6,85 & 4,60 & 1,29 & 3,98 & 4,41 \\
\hline 19 & 5 & 4 & 6,04 & 3,87 & 1,21 & 3,49 & 2,56 \\
\hline 89 & 8 & 4 & 5,17 & 3,29 & 1,08 & 3,66 & 2,22 \\
\hline 110 & 10 & 5 & 5,10 & 3,89 & 1,86 & 4,59 & 3,12 \\
\hline 125 & 24 & 5 & 5,03 & 3,91 & 2,27 & 4,66 & 2,68 \\
\hline 324 & 6 & 4 & 5,46 & 2,89 & 1,10 & 3,19 & 1,90 \\
\hline 592 & 18 & 4 & 4,35 & 2,99 & 1,27 & 3,08 & 1,43 \\
\hline 1982 & 7 & 6 & 5,73 & 4,29 & 1,42 & 3,81 & 2,77 \\
\hline 2671 & 20 & 5 & 5,36 & 3,47 & 1,69 & 3,57 & 2,13 \\
\hline 6902 & 9 & 5 & 6,08 & 3,99 & 1,58 & 4,00 & 2,81 \\
\hline 7521 & 10 & 4 & 5,69 & 3,74 & 1,49 & 3,67 & 2,82 \\
\hline 7810 & 9 & 4 & 5,33 & 3,79 & 1,38 & 3,72 & 2,63 \\
\hline 90044 & 32 & 5 & 5,50 & 4,39 & 2,18 & 4,26 & 3,18 \\
\hline Asturias & 3 & 4 & 4,65 & 3,10 & 1,19 & 3,64 & 1,99 \\
\hline GA & 10 & 7 & 6,45 & 4,91 & 1,49 & 5,07 & 4,69 \\
\hline GB & 11 & 7 & 7,34 & 5,92 & 1,42 & 5,83 & 6,04 \\
\hline GC & 14 & 7 & 6,87 & 5,49 & 1,30 & 5,83 & 5,26 \\
\hline HS-CHR151 & 4 & 6 & 5,31 & 2,84 & 1,12 & 3,28 & 2,49 \\
\hline Marigoule & 20 & 6 & 6,15 & 3,63 & 1,41 & 4,01 & 3,41 \\
\hline Marsol & 5 & 6 & 4,29 & 2,96 & 2,01 & 3,93 & 1,18 \\
\hline
\end{tabular}

Nota: GA, GB y GC corresponden a grupos de clones sin determinar.

La evaluación de los resultados se realizó mediante tres estadísticos numéricos: coeficiente de determinación $\left(R^{2}\right)$, raíz del error medio cuadrático $\left(R E M C, \mathrm{~kg}\right.$ árbol $\left.^{-1}\right)$ y sesgo $\left(E, \mathrm{~kg} \mathrm{rbol}^{-1}\right)$, y a la vez se realizó el análisis gráfico de residuos frente a valores predichos y de valores observados frente a valores predichos.

Se decidió elaborar una única ecuación o tarifa de biomasa conjunta para todos los clones, puesto que el número de pies medidos de cada clon fue muy variable (debido a su disponibilidad en el vivero) y, en la mayoría de los casos, insuficiente para realizar ajustes estadísticos significativos (Tab. 1).

Para establecer los rankings de clones, se definieron inicialmente algunas de las variables de interés según los diferentes objetivos planteados. Así, para conocer qué clones presentan una gran ramificación (lo que influye en la capacidad de producir fruto), se seleccionó la relación entre la biomasa de ramas $\left(w_{r}\right)$ y tres variables control: diámetro en la base $\left(d_{b}\right)$, diámetro normal $\left(d_{n}\right)$ y edad $(t)$. Por otra parte, respecto a la capacidad para uso forestal maderable, se seleccionó la relación de la biomasa en el fuste $\left(w_{f}\right)$ frente a las tres mismas variables de control anteriores (para estudiar la capacidad de producción de madera), y además también se estudió el crecimiento anual empleando las variables diámetro normal $\left(d_{n}\right)$ y altura total $\left(h_{t}\right)$ frente a 
la edad $(t)$. De todas estas características se tomó el criterio de la media aritmética más alta para establecer un orden de clasificación. No obstante, los valores numéricos fueron evaluados conjuntamente con gráficos boxplot para comprobar la variabilidad asociada a cada media.

Resulta evidente que para evaluar las aptitudes anteriores sería preciso analizar también otras variables (por ejemplo, la capacidad de fructificación y el calibre de las castañas, en el caso de la aptitud para fruto), que no es posible estudiar con la muestra utilizada en este trabajo.

\section{Resultados y discusión}

\subsection{Ecuación de biomasa total en peso seco}

El análisis inicial de variables mediante regresión por pasos para elaborar una tarifa de biomasa total en peso seco ( $w_{t o t}, \mathrm{~kg}$ ) para árboles de pequeñas dimensiones indicó que el mejor modelo debía incluir las variables $d_{b}(\mathrm{~cm}), d_{n}(\mathrm{~cm})$ y $h_{t}(\mathrm{~m})$, con un valor de IC $=27.56(\mathrm{IC}<30)$. No obstante, la inclusión de $h_{t}$ además de $d_{b}$ y $d_{n}$ apenas produjo mejora del $R E M C$ y, por tanto, fue descartada. Este resultado contrasta con los obtenidos para otras especies, en las que la altura total sí es la mejor variable, junto con el diámetro normal, para la estimación de biomasa (Diéguez-Aranda et al., 2009).

Todos los parámetros del modelo fueron significativos al 5\%, pero se detectaron ligeros problemas de sobrestimación y heterocedasticidad, que fueron eliminados tras la regresión ponderada (pesos $\left.=1 /\left(d_{n} \cdot d_{b}\right)^{1.698}\right)$. En la Tab. 2 se muestran los resultados del ajuste de la tarifa, indicando los parámetros y los estadísticos del ajuste.

Tabla 2. Resultados del ajuste del modelo de biomasa total en peso seco ( $\left.w_{\text {tot }}, \mathrm{kg}\right)$.

\begin{tabular}{|c|c|c|c|c|c|c|}
\hline Modelo & \multicolumn{3}{|c|}{ Parámetros } & \multicolumn{3}{c|}{ Parámetros } \\
\hline \multirow{2}{*}{ wtot $_{2} a_{0} \cdot d_{b}^{a_{1}} \cdot d_{n}^{a_{2}}$} & $a_{0}$ & $a_{1}$ & $a_{2}$ & $R^{2}$ & REMC & $E$ \\
\cline { 2 - 7 } & $\begin{array}{c}0.05171 \\
(<0.001)\end{array}$ & $\begin{array}{c}1.738 \\
(<0.001)\end{array}$ & $\begin{array}{c}0.7319 \\
(<0.001)\end{array}$ & 0.87 & 0.62 & 0.002 \\
\hline
\end{tabular}

El valor entre paréntesis indica el nivel de significación del parámetro.

\subsection{Rankings de clones}

En la Tab. 3 se presentan los resultados de la clasificación de clones según su mayor aptitud para los objetivos planteados (clones que obtuvieron valores medios superiores a la media de toda la muestra). Estos resultados deben considerarse parciales debido a que, como ya se ha comentado, el número de individuos medidos de cada 
clon fue muy variable y, en muchos casos, insuficiente para realizar ajustes estadísticos significativos; además, también hay que considerar que la edad de los pies estudiados, que osciló entre los 4 y los 7 años (Tab. 1), está todavía por debajo incluso de las edades de corta recomendadas para plantaciones de turno corto de la especie (Cisneros et al., 2008).

Tabla 3. Rankings de los seis mejores clones según objetivos.

\begin{tabular}{|c|c|c|c|c|c|c|c|}
\hline \multirow{2}{*}{ Objetivo } & \multirow{2}{*}{ Variables } & \multicolumn{6}{|c|}{ Mejores clones } \\
\hline & & $1^{\mathrm{o}}$ & $2^{\circ}$ & $3^{\circ}$ & $4^{\circ}$ & $5^{\circ}$ & $6^{\circ}$ \\
\hline \multirow{3}{*}{$\begin{array}{c}\text { Mayor } \\
\text { ramificación }\end{array}$} & $\begin{array}{c}w_{r} \text { vs } d_{n} \\
\left(\mathrm{~kg} \mathrm{~cm}^{-1}\right)\end{array}$ & $\begin{array}{c}3 \\
(0.68)\end{array}$ & $\begin{array}{c}\text { CHR151 } \\
(0.63)\end{array}$ & $\begin{array}{c}\text { Marigoule } \\
(0.61)\end{array}$ & $\begin{array}{c}19 \\
(0.48)\end{array}$ & $\begin{array}{c}89 \\
(0.47)\end{array}$ & $\begin{array}{c}110 \\
(0.47)\end{array}$ \\
\hline & $\begin{array}{c}w_{r} \text { vs } d_{b} \\
\left(\mathrm{~kg} \mathrm{~cm}^{-1}\right)\end{array}$ & $\begin{array}{c}3 \\
(0.45)\end{array}$ & $\begin{array}{c}\text { Marigoule } \\
(0.36)\end{array}$ & $\begin{array}{c}110 \\
(0.35)\end{array}$ & $\begin{array}{c}\text { CHR151 } \\
(0.33)\end{array}$ & $\begin{array}{c}89 \\
(0.30)\end{array}$ & $\begin{array}{l}7810 \\
(0.29)\end{array}$ \\
\hline & $\begin{array}{c}w_{r} \text { vs } t \\
(\mathrm{~kg} \text { año-1 })\end{array}$ & $\begin{array}{c}3 \\
(0.52)\end{array}$ & $\begin{array}{c}19 \\
(0.43)\end{array}$ & $\begin{array}{c}7810 \\
(0.43)\end{array}$ & $\begin{array}{c}7521 \\
(0.42)\end{array}$ & $\begin{array}{c}89 \\
(0.39)\end{array}$ & $\begin{array}{c}\text { Marigoule } \\
(0.39)\end{array}$ \\
\hline \multirow{3}{*}{$\begin{array}{c}\text { Mayor } \\
\text { producción } \\
\text { de madera } \\
\text { en fuste }\end{array}$} & $\begin{array}{c}w_{f} \operatorname{Vs} d_{n} \\
\left(\mathrm{~kg} \mathrm{~cm}^{-1}\right)\end{array}$ & $\begin{array}{l}90044 \\
(0.35)\end{array}$ & $\begin{array}{c}125 \\
(0.33)\end{array}$ & $\begin{array}{c}6902 \\
(0.32)\end{array}$ & $\begin{array}{c}2671 \\
(0.31)\end{array}$ & $\begin{array}{c}7521 \\
(0.31)\end{array}$ & $\begin{array}{c}110 \\
(0.30)\end{array}$ \\
\hline & $\begin{array}{c}w_{f} \text { vs } d_{b} \\
\left(\mathrm{~kg} \mathrm{~cm}^{-1}\right)\end{array}$ & $\begin{array}{l}90044 \\
(0.28)\end{array}$ & $\begin{array}{c}125 \\
(0.26)\end{array}$ & $\begin{array}{c}110 \\
(0.23)\end{array}$ & $\begin{array}{c}6902 \\
(0.21)\end{array}$ & $\begin{array}{c}7521 \\
(0.20)\end{array}$ & $\begin{array}{l}2671 \\
(0.20)\end{array}$ \\
\hline & $\begin{array}{c}w_{f} \text { vs } t \\
\left(\mathrm{~kg} \mathrm{año}^{-1}\right)\end{array}$ & $\begin{array}{l}90044 \\
(0.32)\end{array}$ & $\begin{array}{c}7521 \\
(0.29)\end{array}$ & $\begin{array}{c}125 \\
(0.26)\end{array}$ & $\begin{array}{c}6902 \\
(0.25)\end{array}$ & $\begin{array}{l}7810 \\
(0.23)\end{array}$ & $\begin{array}{c}110 \\
(0.23)\end{array}$ \\
\hline \multirow{2}{*}{$\begin{array}{c}\text { Mayor } \\
\text { crecimiento } \\
\text { en diámetro } \\
\text { y altura }\end{array}$} & $\begin{array}{c}d_{n} \text { vs } t \\
\left(\mathrm{~cm} \text { año }{ }^{-1}\right)\end{array}$ & $\begin{array}{c}19 \\
(0.97)\end{array}$ & $\begin{array}{c}7810 \\
(0.95)\end{array}$ & $\begin{array}{l}7521 \\
(0.94)\end{array}$ & $\begin{array}{l}90044 \\
(0.88)\end{array}$ & $\begin{array}{c}89 \\
(0.82)\end{array}$ & $\begin{array}{c}6902 \\
(0.80)\end{array}$ \\
\hline & $\begin{array}{c}h_{t} \text { vs } t \\
(\mathrm{~m} \text { año }\end{array}$ & $\begin{array}{c}7810 \\
(0.93)\end{array}$ & $\begin{array}{c}110 \\
(0.92)\end{array}$ & $\begin{array}{l}7521 \\
(0.92)\end{array}$ & $\begin{array}{c}125 \\
(0.92)\end{array}$ & $\begin{array}{c}89 \\
(0.92)\end{array}$ & $\begin{array}{c}\text { Asturias } \\
(0.91)\end{array}$ \\
\hline
\end{tabular}

Nota: El valor entre paréntesis indica el valor de la variable considerada.

Como puede observarse, en cuanto a la capacidad de ramificación, el clon 3 presentó la mayor biomasa de ramas respecto a todas las variables de control. Sin embargo, su fruto es pequeño (Fernández-López y Miranda, 2007), y es un árbol de altura y grosor medios. Otros clones que destacan en este apartado son el Marigoule, el 19, el CHR151, el 89 y el 110.

Para la producción de biomasa de fuste destaca el clon 90044, que presentó los mayores valores respecto a todas las variables de control. A continuación le seguirían los clones 125, 6902, 7521 y 110 . El clon 125, además de presentar mucha biomasa en el fuste, posee un fruto grande (Fernández-López y Miranda, 2007), por lo que puede cumplir con diferentes objetivos.

El clon 7810 presentó el mejor crecimiento anual en altura, y además el segundo mejor crecimiento anual en diámetro, por lo que destaca en este apartado, aunque so- 
lamente aparece una vez en los rankings de mayor producción de madera de fuste (en $5^{\text {a }}$ posición). El clon 19 fue el que mostró un mayor crecimiento anual en diámetro, pero no aparece entre los seis mejores en crecimiento en altura ni en producción de madera de fuste (aunque sí segundo en producción anual de biomasa de ramas). Sin embargo, el clon 7521, tercero en los crecimientos anuales en diámetro y altura, sí se sitúa entre los mejores en cuanto a producción de madera de fuste, por lo que se puede considerar como uno de los mejores para uso forestal maderable. Otra ventaja de los clones 7521 y 7821 es que son resistentes a la tinta.

El clon 89 no es el mejor en ninguna categoría, pero está presente entre los de mayor crecimiento de ramificación, de diámetro y altura, por lo que parece un clon de gran porte adecuado para la producción de castaña, cuyo tamaño además es muy grande (Fernández-López y Miranda, 2007).

De los clones estudiados por Álvarez-Álvarez (2004) que también se incluyeron en este trabajo, los resultados parecen coincidir en su mayoría, excepto para los clones 3 y 90044, propuestos como de uso forestal y de fruto respectivamente por dicho autor, al contrario de lo observado en esta ocasión. Estas disparidades pueden ser debidas a que en el presente trabajo se ha considerado exclusivamente la capacidad de ramificación como criterio para definir la aptitud o vocación de los clones para fruto, y no otros como la capacidad de fructificación o el tamaño de las castañas (imposibles de determinar con la información disponible para este trabajo).

\section{Conclusiones}

Se ha ajustado una ecuación para la estimación de la biomasa total en seco de árboles de pequeñas dimensiones (conjunta para todos los clones estudiados), que ha incluido las variables diámetro en la base $\left(d_{b}\right)$ y diámetro normal $\left(d_{n}\right)$, pero no la altura total de los árboles $\left(h_{t}\right)$.

Se han establecido unos rankings de clones en función de sus aptitudes. Los resultados indican que los clones 3, 19, CHR151, 89 y 110 serían los más adecuados para fruto (considerando exclusivamente su mayor producción de ramas), mientras que los clones $90044,125,110,7521$ y 7810 lo serían para producción de madera. Estos resultados deben considerarse parciales debido al escaso número de pies disponibles para muchos de los clones estudiados.

\section{Agradecimientos}

Este trabajo fue financiado parcialmente mediante contratos de investigación con la empresa BOIBEL FORESTAL S.L., dentro de los proyectos:

- "SOUTONOVO: cooperación para o desenvolvemento dun novo proceso integrado de talladía do castiñeiro" (2008-2010, "Accións de cooperación para o desenvolvemento de novos produtos, procesos e tecnoloxías no ámbito agro- 
alimentario, agrícola e forestal da Consellería do Medio Rural", Xunta de Galicia).

- "INTEGRACASTANEA: Proxecto de integración vertical do castiñeiro: da mellora xenética ás producións finais" (2013-2014, programa FEDER-INNTERCONECTA II Galicia).

La "Unidade de Xestión Forestal Sostible" (UXFS) está financiada por la Consellería de Cultura, Educación e Ordenación Universitaria de la Xunta de Galicia mediante el "Programa de Consolidación e Estructuración de Unidades de Investigación Competitivas, modalidade de Grupos de Referencia Competitiva", para el período 2014-2017.

\section{Bibliografía}

Álvarez-Álvarez, P. 2004. Viveros forestales y uso de planta en repoblación en Galicia. Tesis doctoral. Universidad de Santiago de Compostela.

Álvarez-Álvarez, P., Barrio Anta, M., Castedo Dorado, F., Díaz Varela, R.A., Fernández Lorenzo, J.L., Mansilla Vázquez, P., Pérez Otero, R., Pintos Varela, C., Riesco Muñoz, G., Rodríguez Soalleiro, R.J., Salinero Corral, M.C. 2000. Manual de selvicultura del castaño en Galicia. Universidad de Santiago de Compostela. 120 pp.

Belsley, D.A. 1991. Conditioning diagnostics, collinearity and weak data in regression. John Wiley \& Sons, Inc., New York.

Cisneros, O., Cabrera, M., Montero, G. 2008. Selvicultura de Castanea sativa Mill. In: Serrada, R., Montero, G., Reque, J.A. (eds.), Compendio de selvicultura aplicada en España. Instituto Nacional de Investigación y Tecnología Agraria y Alimentaria, Madrid, pp. $83-116$.

Diéguez-Aranda, U., Rojo-Alboreca, A., Castedo-Dorado, F., Álvarez González, J. G., BarrioAnta, M., Crecente-Campo, F., González González, J. M., Pérez-Cruzado, C., Rodríguez Soalleiro, R., López-Sánchez, C. A., Balboa-Murias, M. A., Gorgoso Varela, J. J., Sánchez Rodríguez, F. 2009. Herramientas selvicolas para la gestión forestal sostenible en Galicia. Consellería do Medio Rural, Xunta de Galicia. 268 pp.

Fernández-López, J., Alía, R. 2008. EUFORGEN Guía técnica para la conservación genética y utilización del castaño (Castanea sativa). Foresta. Madrid. España.

Fernández-López, J., Miranda, M.E. 2007. Materiais de base de clons de castiñeiro híbrido para a producción de madeira (Castanea crenata $\mathrm{x}$ Castanea sativa). Tríptico editado por la Consellería de Medio Rural, Dirección Xeral de Investigación, Tecnología e Formación Agroforestal. Xunta de Galicia.

Myers, R. H. 1986. Classical and modern regression with applications. Duxbury Press, Boston, Masssachusetts. 488 pp.

R Core Team. 2014. R, A language and environment for statistical computing. R Foundation for Statistical Computing, Vienna, Austria. ISBN 3-900051-07-0, URL: http,//www.Rproject.org/

Schumacher, F. X., Hall, F. S. 1933. Logarithmic expression of timber-tree volume. Journal of Agricultural Research 47, 719-73. 
Xunta de Galicia. 2007. Orde do 16 de abril de 2007 pola que se aproba a delimitación e a determinación dos materiais de base para a producción de materiais forestais de reproducción de Acer pseudoplatanus L., Fraxinus excelsior L., Pinus sylvestris L., Pseudotsuga menziesii Franco, Prunus avium L., Pinus pinaster Ait., e híbridos artificiais de Castanea spp. no territorio da Comunidade Autónoma de Galicia (DOGA n ${ }^{\circ}$ 77, 20 de abril de 2007). 
